\title{
Autonomous 3D geometry reconstruction through robot-manipulated optical sensors
}

Carmelo Mineo ( $\square$ carmelo.mineo01@unipa.it)

Università degli Studi di Palermo https://orcid.org/0000-0002-5086-366X

Donatella Cerniglia

University of Palermo

Vito Ricotta

University of Palermo

Bernhard Reitinger

University of Palermo

\section{Research Article}

Keywords: View planning, 3D reconstruction, Adaptive mapping, Metrology, Inspection, Robotics

Posted Date: April 8th, 2021

DOI: https://doi.org/10.21203/rs.3.rs-399855/v1

License: (c) (1) This work is licensed under a Creative Commons Attribution 4.0 International License. Read Full License

Version of Record: A version of this preprint was published at The International Journal of Advanced Manufacturing Technology on July 5th, 2021. See the published version at https://doi.org/10.1007/s00170-021-07432-5. 


\section{Abstract}

Many industrial sectors face increasing production demands and need to reduce costs, without compromising the quality. Whereas mass production relies on well-established protocols, small production facilities with small lot sizes struggle to update their highly changeable production at reasonable costs. The use of robotics and automation has grown significantly in recent years, but extremely versatile robotic manipulators are still not commonly used in small factories. Beside of the investments required to enable efficient and profitable use of robot technology, the efforts needed to program robots are only economically viable in case of large lot sizes. Generating robot programs for specific manufacturing tasks still relies on programming trajectory waypoints by hand. The use of virtual simulation software and the availability of the specimen digital models can facilitate robot programming. Nevertheless, in many cases, the virtual models are not available or there are unavoidable differences between virtual and real setups, leading to inaccurate robot programs and time-consuming manual corrections. This could be avoided by measuring the real-geometry and the position of the specimen, which creates the paradox of having to plan robot paths for surface mapping purposes, before the originally intended robot task can be approached. Previous works have demonstrated the use of robotically manipulated optical sensors to map the geometry of samples. However, the use of simple user-defined robot paths, which are not optimized to the part geometry, typically causes some areas of the samples to not be mapped with the required level of accuracy or to not be sampled at all by the optical sensor. This work presents an autonomous framework to enable adaptive surface mapping, without any previous knowledge of the part geometry being transferred to the system. The article gives an overview of the related work in the field, a detailed description of the proposed framework and a proof of its functionality through both simulated and experimental evidences.

\section{Full Text}

Due to technical limitations, full-text HTML conversion of this manuscript could not be completed. However, the manuscript can be downloaded and accessed as a PDF.

\section{Figures}


(a)

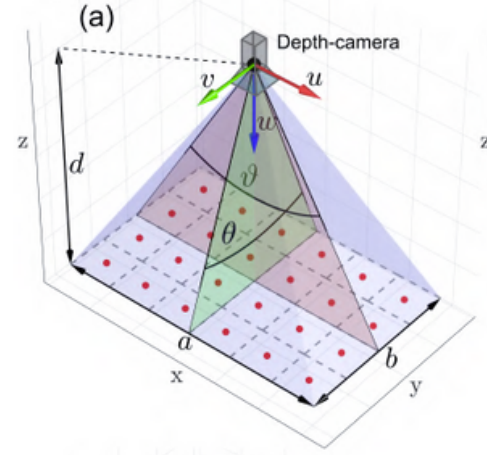

(b)

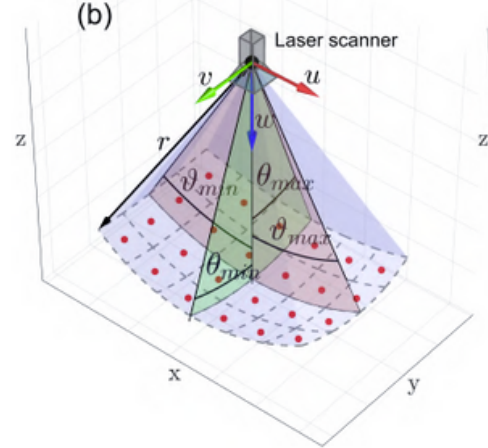

(c)

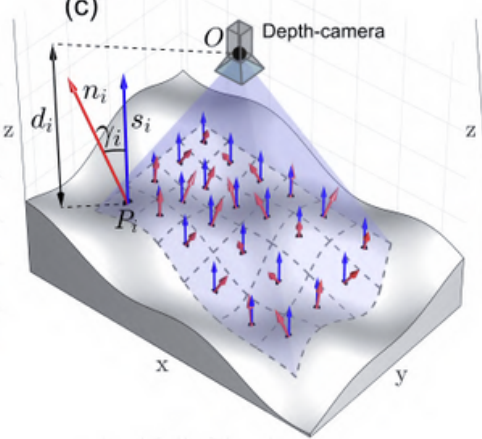

(d)

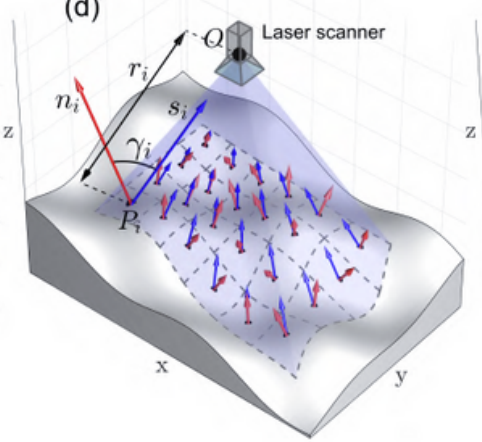

(e)

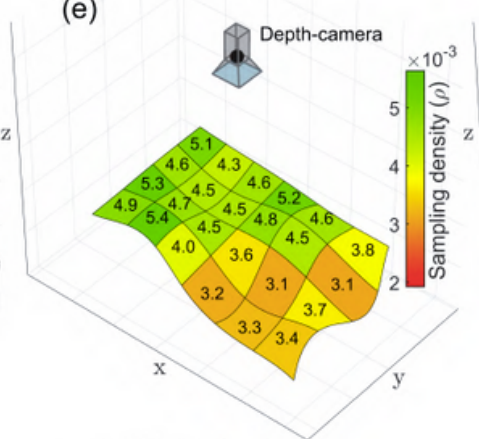

(f)

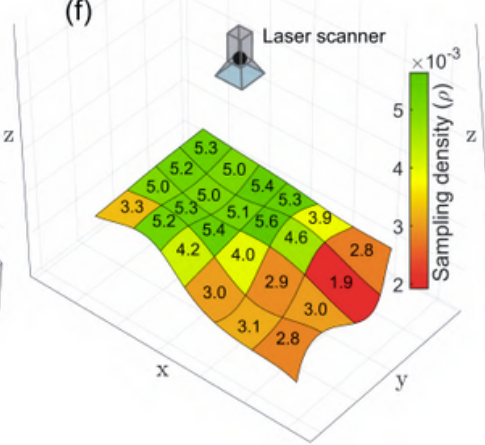

(g)

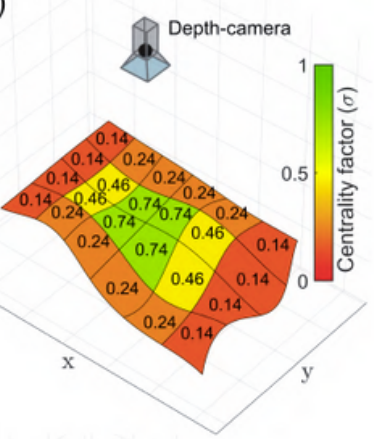

(h)

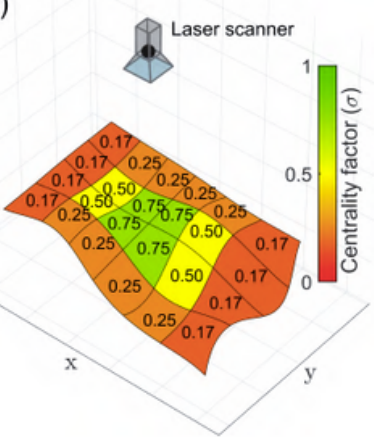

Figure 1

Fundamental working parameters for a depth-camera sensor (a) and a laser scanner sensor (b), representation of the vectors for the computation of the local sampling density on an example surface (cd), local sampling densities (e-f) and centrality factors ( $g-h)$ computed for all points collected by the generic depth-camera and laser scanner. 

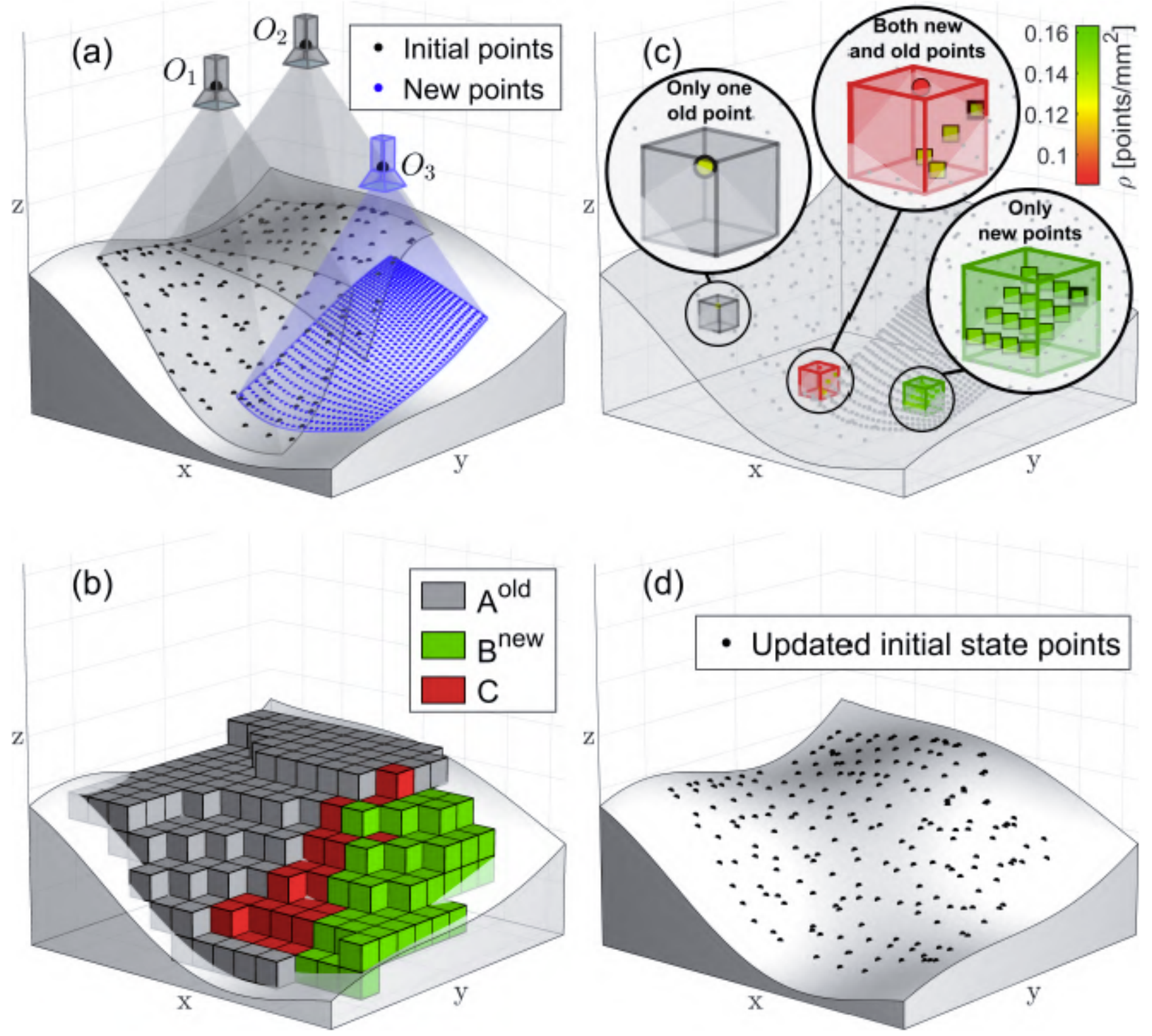

Figure 2

Initial state points and new incoming points (a). Grouping points into cubes of side equal to the target sampling density (b). Example of selection of maximum sampling density point in a cube containing only one old point, only new points and both old and new points (c). Resulting merged and down-sampled new initial state $(d)$. 

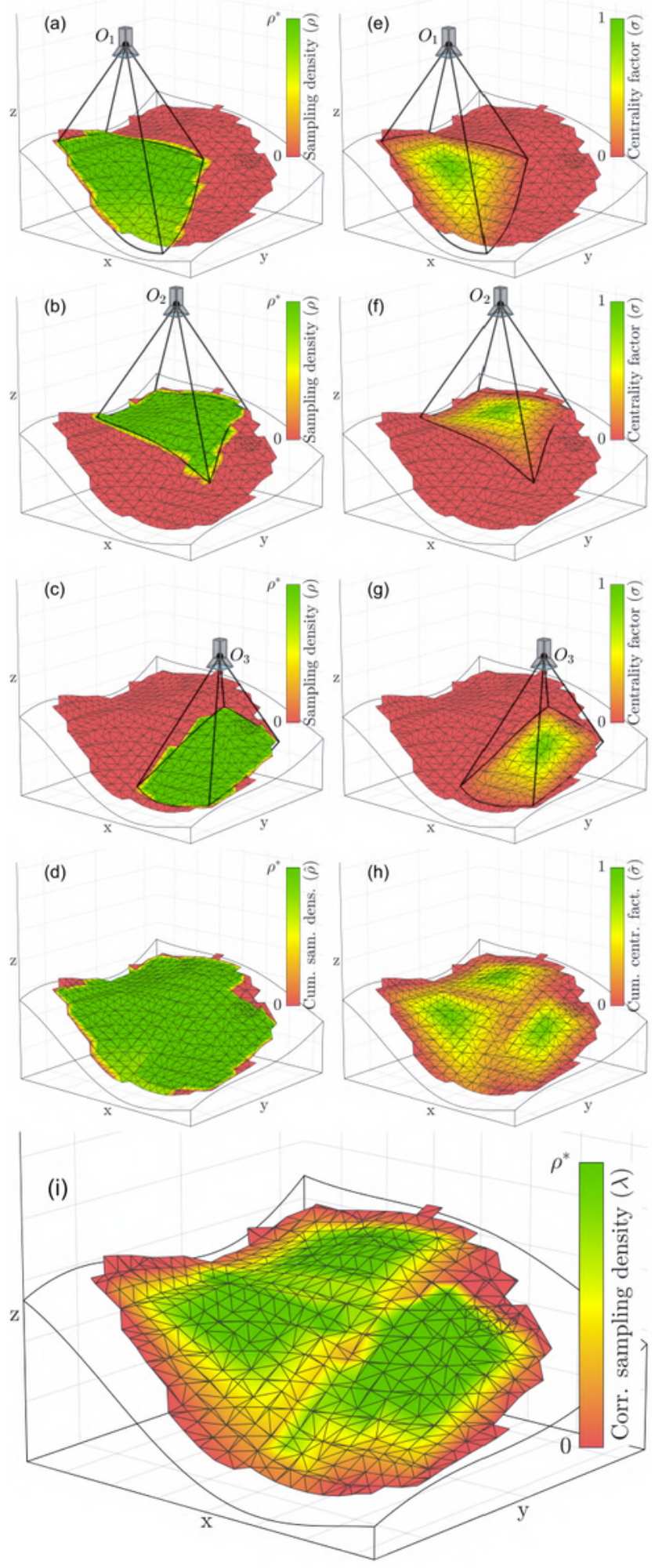

\section{Figure 3}

Sampling density relative to each sensor pose (a-c), cumulative sampling distance (d), centrality factor relative to each sensor pose (e-g), cumulative centrality factor (h) and corrected cumulative sampling density (i). 

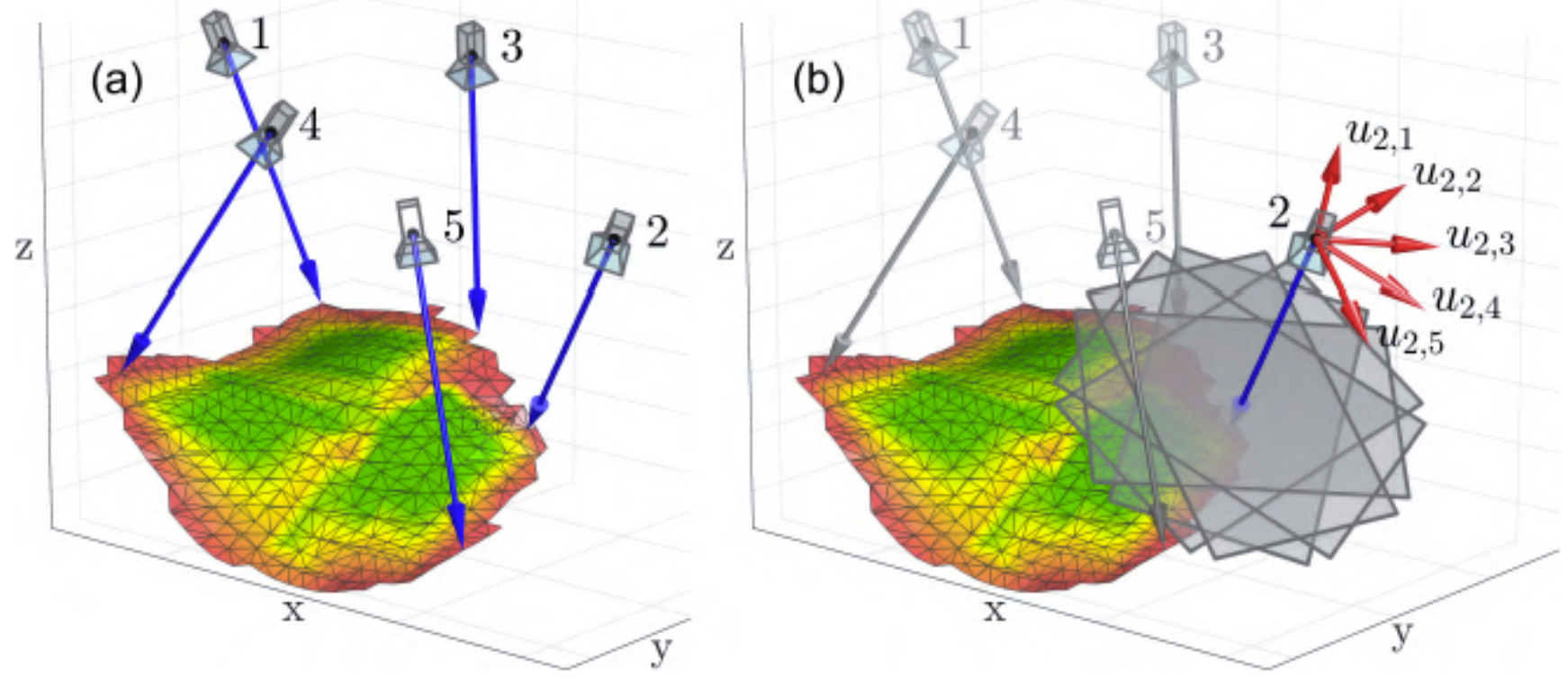

\section{Figure 4}

The rst ve test view point and direction (a) and illustration of the selection of four sensor orientations for each selected test direction (b). 

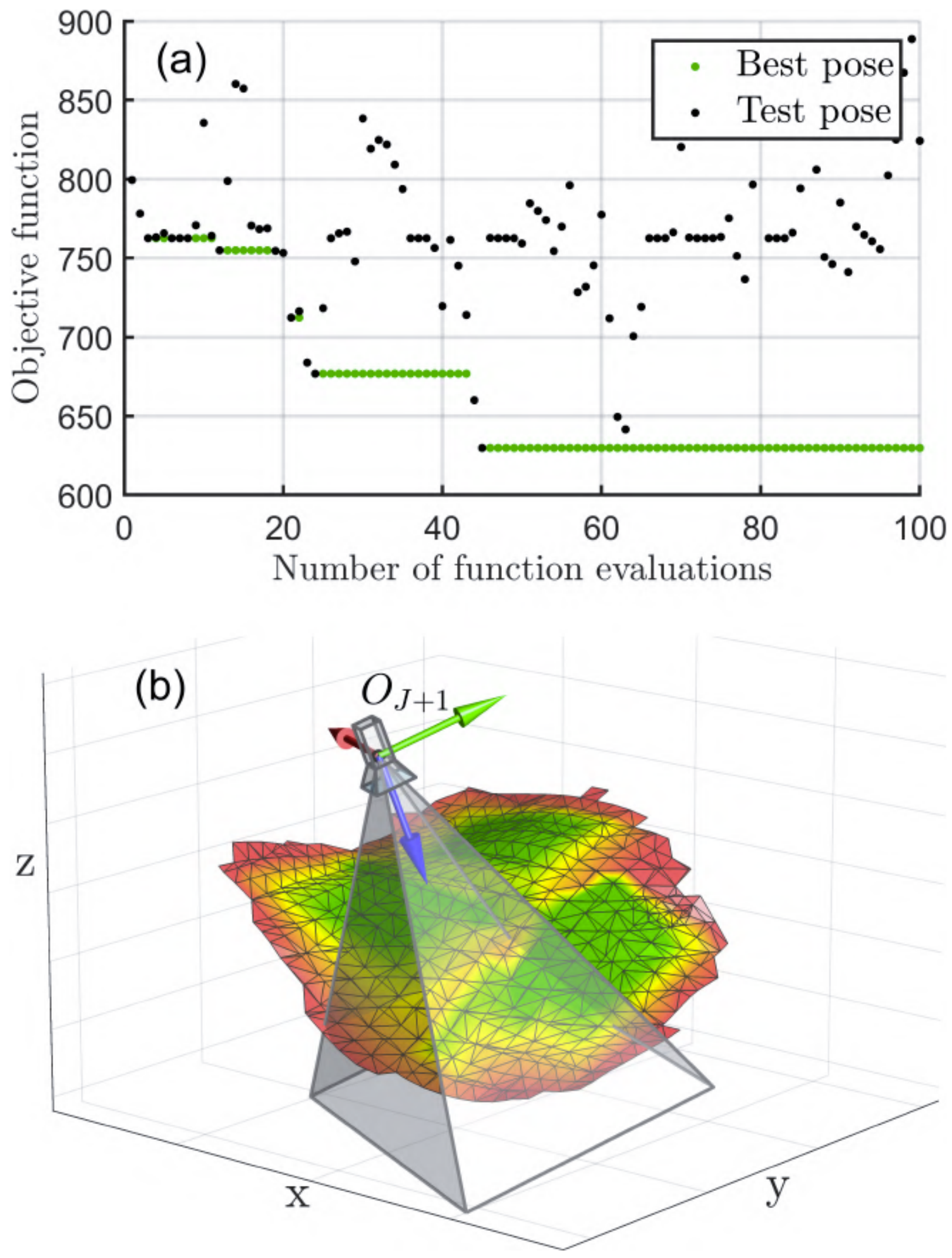

Figure 5

Evaluation of the objective function value at the test poses (a) and illustration of the determined next best pose for the given example (b). 

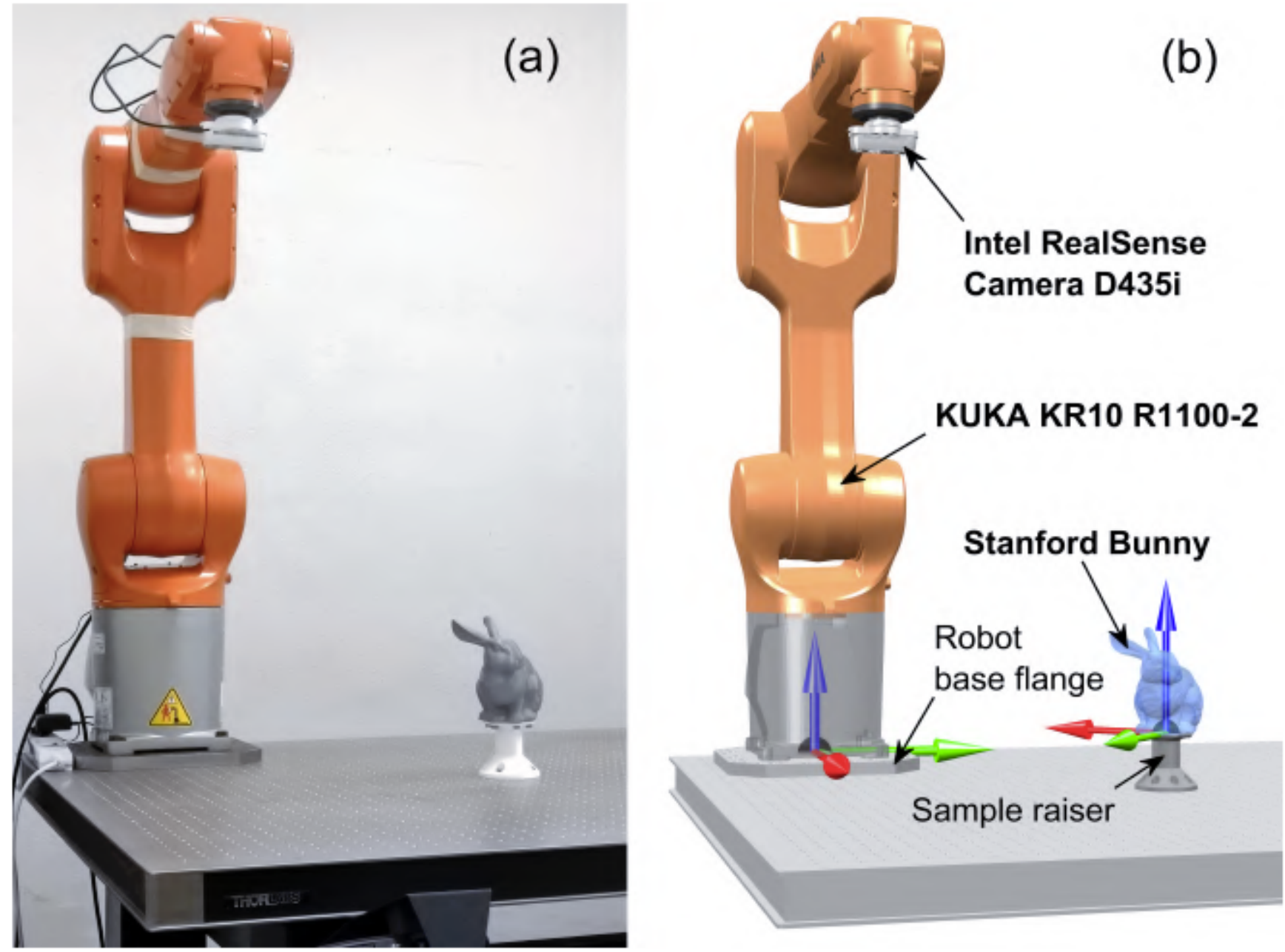

Figure 6

Real (a) and virtual (b) experimental setup, showing the Intel ${ }^{\circledR}$ RealSense ${ }^{\mathrm{TM}}$ Depth Camera D435i, mounted onto the KUKA KR10-R1100-2 robot, and the 3D printed Stanford Bunny test model. 

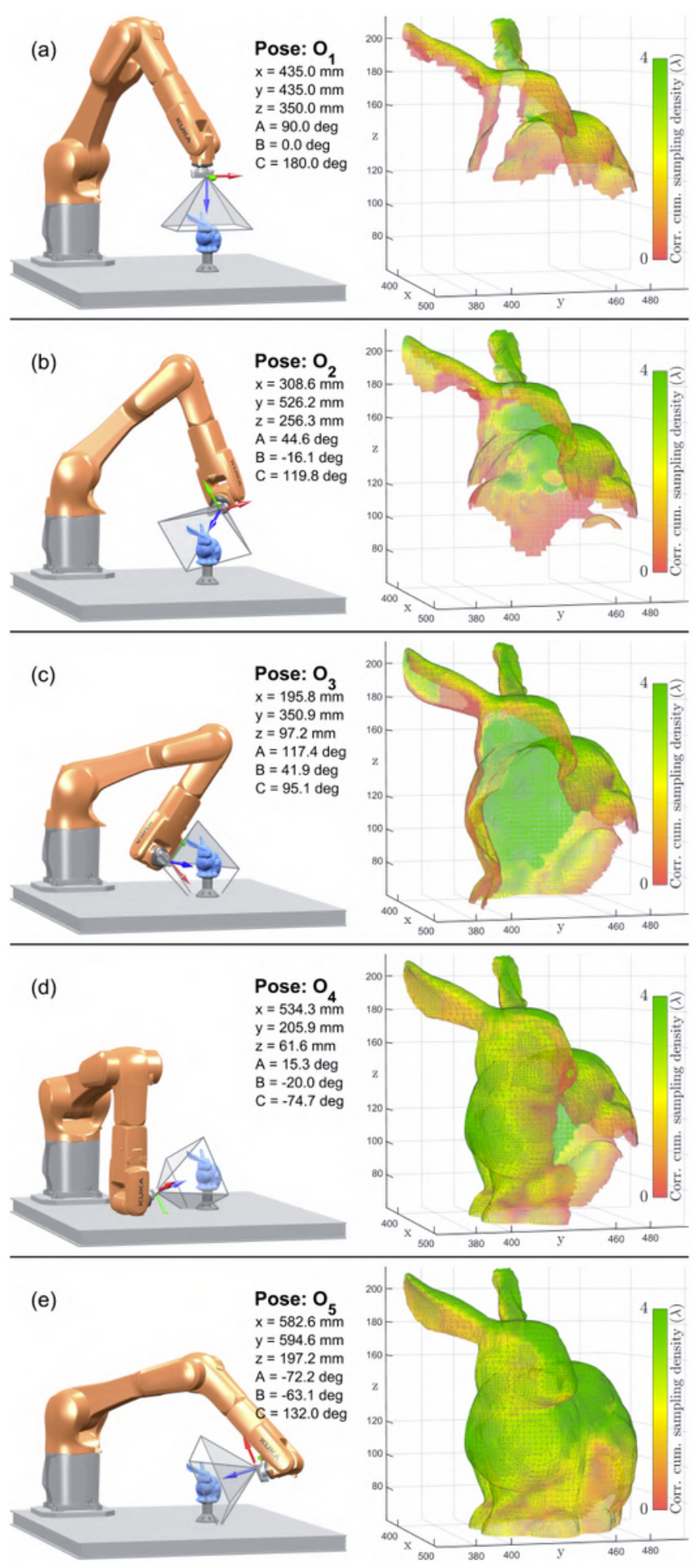

\section{Figure 7}

Simulated full 3D reconstruction of Stanford Bunny with target density $\mathrm{P}^{\star}=0.05$ points $/ \mathrm{mm} 2$, through an initial starting pose (a) and four autonomously generated sensor poses (b-e). 


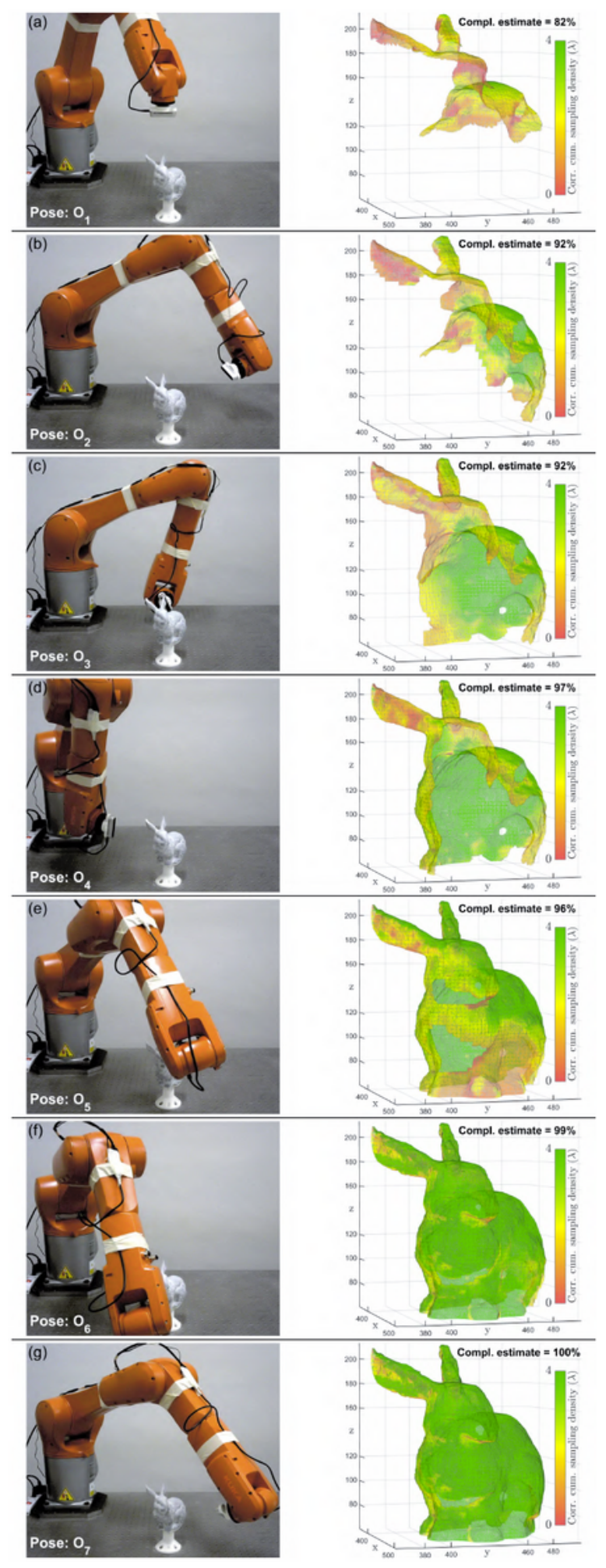

\section{Figure 8}

Full 3D reconstruction of Stanford Bunny with target density $\mathrm{P}^{*}=0.05$ points $/ \mathrm{mm} 2$, through an initial starting pose (a) and six autonomously generated sensor poses (b-g). 


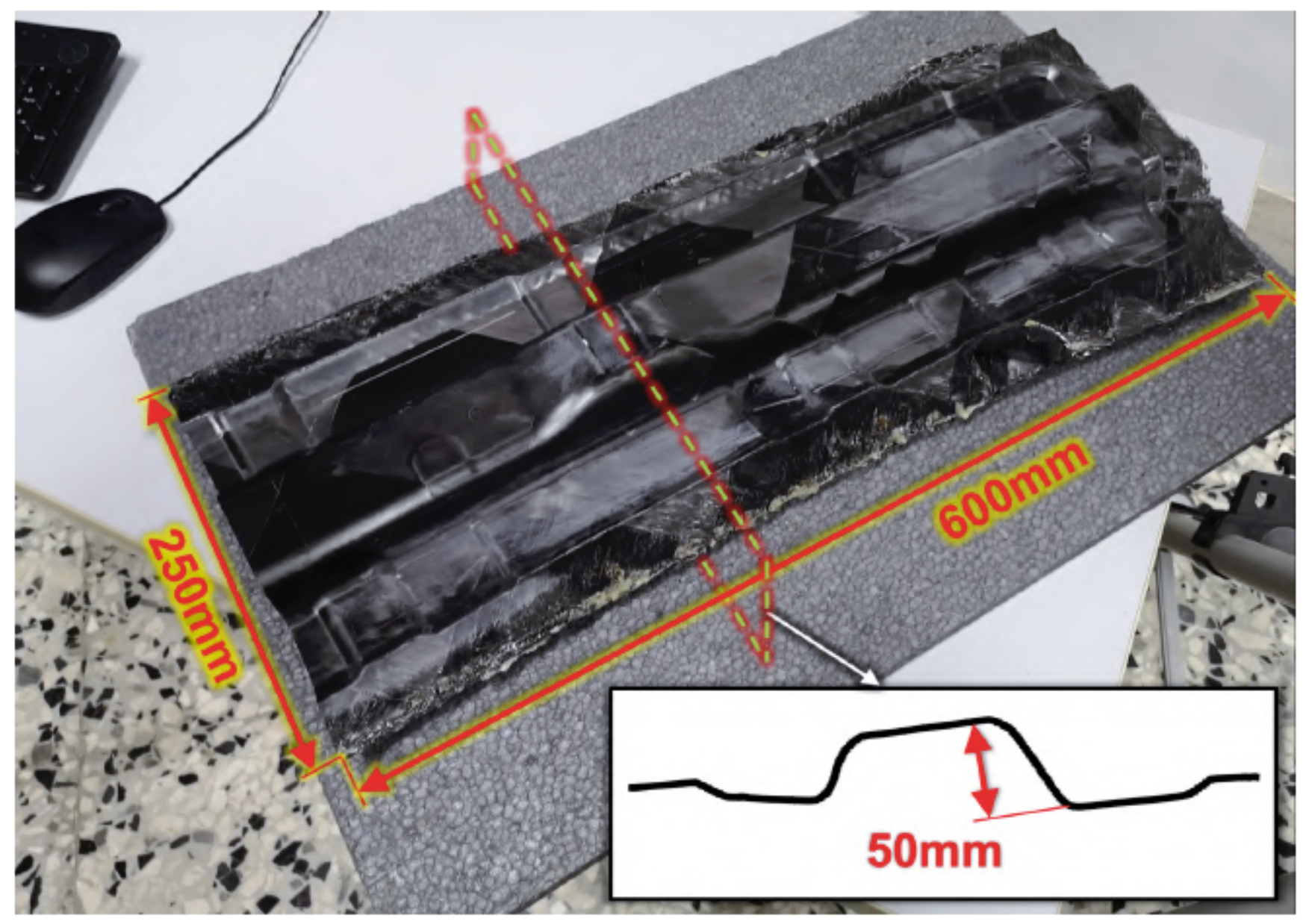

Figure 9

CFRP automotive sample used as additional test case. 

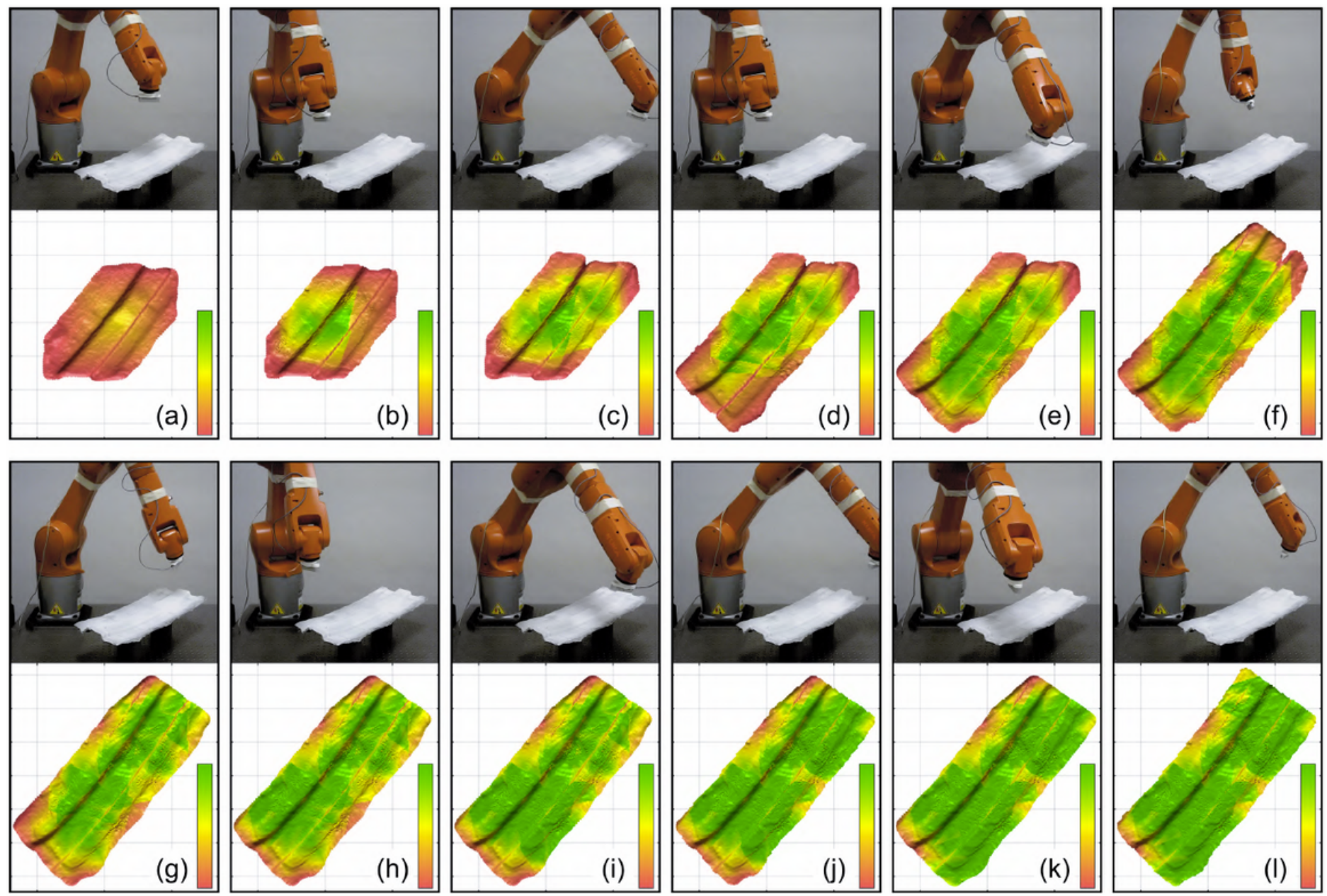

\section{Figure 10}

Full 3D reconstruction of CFRP automotive test sample with target density $\mathrm{P}^{\star}=0.05$ points $/ \mathrm{mm} 2$, through an initial starting pose (a) and eleven autonomously generated sensor poses (b-l). 

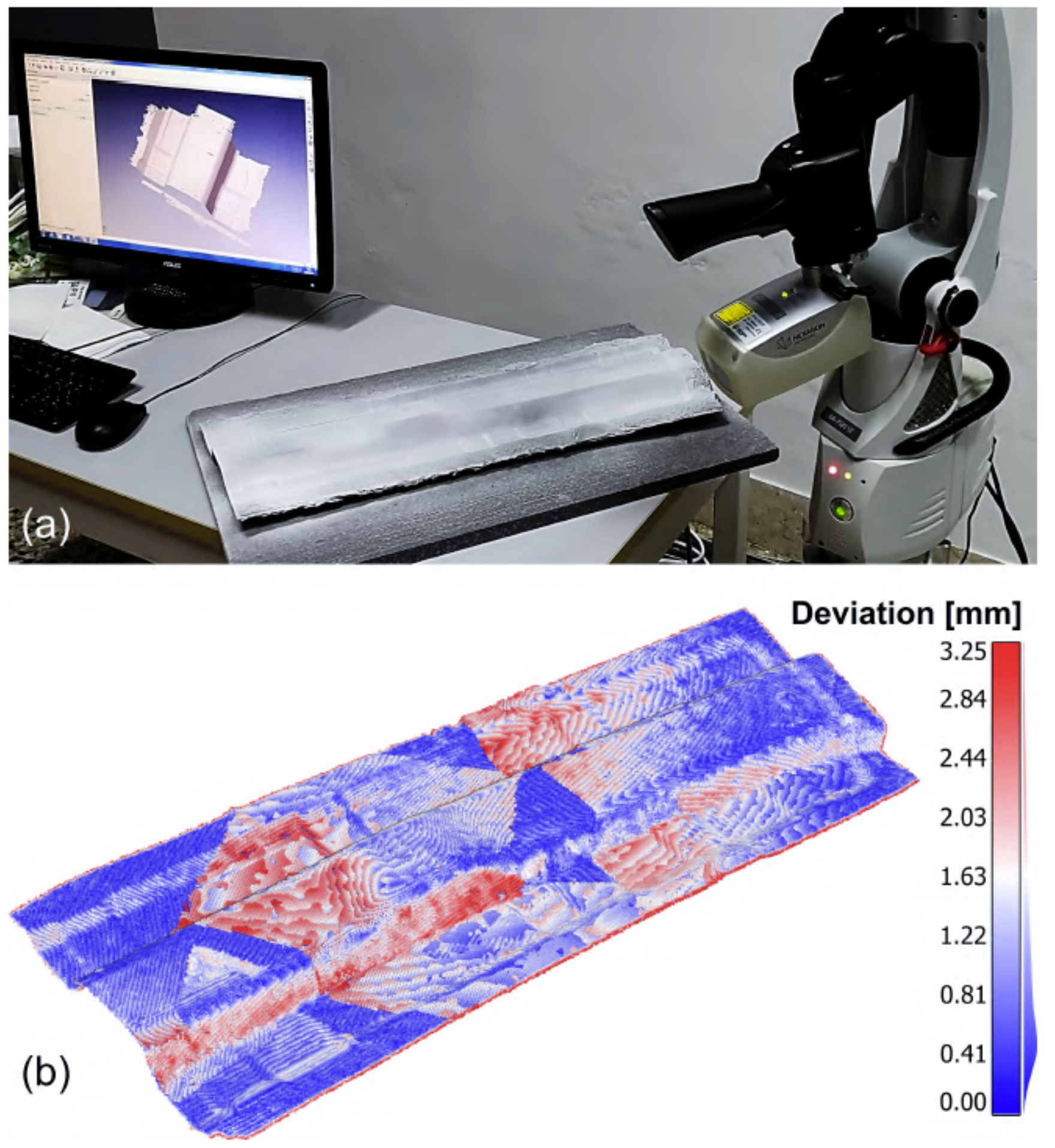

Figure 11

Acquisition of ground-truth point cloud through the Hexagon ROMER Absolute Arm (a) and map of deviation between the reconstructed geometry and the ground-truth (b). 